\title{
THE MAGNETOSPHERES OF THE HELIUM PECULIAR STARS OF THE UPPER MAIN SEQUENCE
}

\author{
STEVEN N. SHORE \\ GHRS Science Team/Computer Sciences Corporation \\ Goddard Space Flight Center, Code 681, Greenbelt, MD 20771 USA \\ and DEMIRM, Observatoire de Meudon
}

\begin{abstract}
A brief review is presented of the evidence for magnetospheres surrounding the upper main sequence helium peculiar stars. Some of the underlying physical processes are discussed. The helium strong stars all appear to possess comparatively dense magnetospheres. The phenomenon is also present among some helium weak stars, but is quite rare.
\end{abstract}

\section{INTRODUCTION}

The general properties of the helium peculiar stars have been reviewed by Hunger at this conference, and Linsky has discussed the details of their radio emission. I will concentrate here on the general aspects of the magnetospheric environment, the locale where many of the phenomena that make the helium stars unique take place. The helium peculiar stars have provided many interesting puzzles over the years, but perhaps none has been so intriguing as the evidence for trapped circumstellar matter. The first inkling of this phenomenon came with the discovery of Balmer emission line variations in the well known peculiar star $\sigma$ Ori E, also known as HD 37479 (Walborn 1974). This discovery was followed rapidly by the observation of an eclipse-like light curve (Hesser, Walborn, and Ugarte 1976) and the connection of the photometric and emission line variations (Walborn and Hesser 1976). At the same time, Groote and Hunger (1976) discovered variable shell cores in the higher Balmer series lines.

The $\mathrm{H} \alpha$ emission line variations were modeled by Nakajima (1985) using clouds trapped above the magnetic equator in an oblique rotator frame. Similar models have been proposed by Groote and Hunger (1982) and Bolton et al. (1987) for $\sigma$ Ori $\mathrm{E}$, with more complications to account for what appear to be departures from strict axial symmetry in the magnetic frame. Balmer emission line variations have been detected in several of the other helium strong stars, but no specific models have been proposed.

The helium weak stars have been extensively studied, but none shows any emission line variability. 
Radio emission was reported by Drake et al. (1987) and Linsky et al. (1992) (see also Linsky, these proceedings). They attribute the emission to gyrosynchrotron emission in a distended magnetosphere. Phillips and Lestrade (1988) have performed VLBI measurements that resolved the emission region in $\sigma$ Ori $\mathrm{E}$, showing that the emission region is at about 10 stellar radii. They also showed that the emission is nonthermal. Leone and Umana (1992, see Leone, these proceedings) have argued that the emission in HD 37017 and 37479 can be modeled using gyrosynchrotron. In general, however, these pictures take the source of the particles more or less for granted.

From the theoretical side, the first work on stellar magnetospheres appears to have been Deutsch's (1957) solution for the emission of electromagnetic radiation by a rotating magnetized star. He found a rotating magnetized star emits Poynting flux, $\mathbf{E} \times \mathbf{B} / c$, that carries away angular momentum from the star. The same solution was found many years later by Pacini (1967) in his study of rotating magnetized neutron stars, the precursor study for the pulsars, and later still by Goldreich and Julian (1968). The basic result is quite easy to understand. One assumes that the star is a rotating dipole, with a magnetic moment $\mu$ and a rotation frequency $\Omega$. Then the emission rate follows from Larmor's theorem, $d E / d t \sim|\ddot{\mu}|^{2} \sim B^{2} R^{6} \Omega^{6}$, so that the spindown of such a star is given approximately by $\dot{\Omega} \sim \Omega^{5}$. Now the emission occurs for a massless magnetic field, one that connects continuously with a vacuum radiated wave and where there is no problem with a return current. The field is supported by currents inside the star and the vacuum solution is (presumably) force free. How this relates to the Ap stars is seen through the assertion that the rate of spindown depends on the rotation frequency and the dipole moment. However, this model for the spindown treats the star in vacuo, while in reality we expect that the star is surrounded by a (possibly dense) magnetosphere.

\section{STELLAR WINDS AND MAGNETOSPHERES}

First, it is important to note that magnetospheres are actually necessary consequences of rotation, mass loss, and strong magnetic fields. Any ejected material will corotate to some extent out to large distances from the star, at least out to the Alfvén radius. We'll assume that this is the point where the magnetic stress equals the centrifugal inertial term, that is where the energy density in the magnetic field is the same as the kinetic energy of the corotating plasma. We will return to this in a moment, but let's examine the structure of the wind and how a magnetic stellar wind relates to a magnetosphere.

For an isothermal wind, we have:

$$
\nabla \cdot \rho \mathbf{v}\left(\frac{1}{2} v_{p}^{2}+\frac{1}{2} \Omega r^{2} \sin ^{2} \theta+a_{s}^{2} \ln \rho-\frac{G M}{r}\right)=-\nabla \cdot \frac{c}{4 \pi}(\mathbf{E} \times \mathbf{B})
$$

where $\theta$ is the latitude on the rotating star. The lefthand side represents the advected energy flux and the right hand side is the Poynting flux in the magnetic field. This last equation was derived by Mestel (1968). The flow has the steady state solution, where $\nabla \cdot \rho \mathbf{v}=0$ and $\nabla \cdot \mathbf{B}=0$, that $\mathbf{v}=\kappa \mathbf{B}$ where $\kappa$ is a scalar function. This means that the flow suffers a dead zone at the magnetic equator and looks like an extended atmosphere otherwise for a centrifugally driven wind. This dead zone extends out to the Alfvén radius, where it forms an expanding current sheet. 
For a surface dipolar magnetic field, the radius at which the corotation speed is the same as the Alfvén speed is given by :

$$
v_{A}=\frac{B_{0} R_{\star}^{3}}{R_{A}^{3}\left(4 \pi \rho_{A}\right)^{1 / 2}}=R_{A} \Omega
$$

If the mass loss is steady-state, then $\dot{M}=4 \pi R_{A}^{2} \rho_{A} v\left(R_{A}\right)$, where $v$ is the radial velocity. Now we need to make an assumption ( $c f$. Michel 1991) that the radial velocity at the Alfvén point is approximately the same as the terminal velocity, $v\left(R_{A}\right) \approx v_{\infty}$. Thus a good estimate for the radius of the Alfvén surface is:

$$
R_{A} \approx\left(\frac{B_{0}^{2} v_{\infty}}{\dot{M} \Omega^{2}}\right)^{1 / 6} R_{\star} \approx 8.2 \times 10^{-3} B_{0, G}^{1 / 3} V_{\infty}^{1 / 6} \dot{m}^{-1 / 6} P_{d}^{1 / 3} R_{\star},
$$

where the magnetic field is now in Gauss, the mass loss rate is in $M_{\odot} \mathrm{yr}^{-1}$, the terminal velocity is in $\mathrm{km} \mathrm{s} \mathrm{s}^{-1}$, and the rotation period, $P_{d}$, is in days. For a 10 $M_{\odot}$ star with a rotation period of about 2 days, a mass loss rate of $10^{-8} M_{\odot}$ $\mathrm{yr}^{-1}$, and with a strong surface magnetic field of order $5 \mathrm{kG}$, the escape velocity is about $1000 \mathrm{~km} \mathrm{~s}^{-1}$ so that the Alfvén radius is at about 10 stellar radii. Notice that as the mass loss rate decreases, the Alfvén radius moves outward.

The net energy flux for a steady state magnetic wind is given by:

$$
F=\rho v_{r} r^{2}\left[\frac{1}{2} V^{2}+\frac{\gamma}{\gamma-1} \frac{p}{\rho}-\frac{G M}{r}+\frac{B_{r} B_{\phi}}{4 \pi \rho} \frac{\Omega r}{v_{r}}\right]=\text { constant }
$$

The total velocity is $V=\left(v_{r}^{2}+v_{\phi}^{2}\right)^{1 / 2}$, the last term represents the Poynting flux carried by the field with a tilt angle $\Omega r / v_{r}, p$ is the gas pressure, and $\rho$ is the density. Since $\rho v_{r} r^{2}$ is constant, we have an equation that is an algebraic expression of the solution to the equation of motion analogous to the Parker solution. If we choose a particular value of $F / \dot{M}$, we can solve for the radial velocity of the flow with distance:

$\frac{1}{2} v_{A}^{2} M_{A}^{2}+\frac{\gamma}{\gamma-1} \frac{p_{A}}{\rho_{A}} M_{A}^{-2(\gamma-1)}-\frac{G M}{r_{A} x}+\frac{\Omega r_{A}^{2}}{2 x^{2}}\left[1+\frac{\left(2 M_{A}^{2}-1\right)\left(x^{2}-1\right)}{r_{A}^{2}\left(M_{A}^{2}-1\right)}\right]=$ constant

We have the free parameters $v_{A}$ and $r_{A}$ for the quantitative solution. The flow reaches a critical point at the Alfvén radius and proceeds to a large terminal velocity, essentially the escape velocity, transporting angular momentum through the strong coupling afforded by the magnetic field.

Most of the theoretical work has focused on the weak field limit, essentially extensions of the Weber-Davis type solution for fast winds. Nerney and Suess (1987) applied the fast magnetic rotator (Belcher and MacGregor 1976) to the problem, and Shore (1987) treated some of the theory for a radiative stellar wind in a strong magnetic field. But the implications for the magnetospheric environment have not been explored in detail.

The effect of radiation pressure is primarily to alter the gravitational acceleration by replacing it with $g_{\text {eff }}=g(1-\Gamma)$, where $\Gamma$ is the ratio of the radiation to gravitational acceleration (in a more luminous star, this would be the ratio of the Eddington luminosity to the gravitational acceleration). This effect is 
spherically symmetric (allowing for slow rotation so that the star isn't much distorted). The main effects of the field will be to break the spherical symmetry and to suppress the mass loss at the magnetic equator (Shore 1987). The polar solution is essentially the same as for a one dimensional stellar wind the critical point is the sound speed. At the magnetic equator, the transverse plasma flow encounters a resistance by the field that alters the critical point to correspond to the fast magnetoacoustic speed. Thus a polar wind is produced by the freely streaming plasma that moves along open field lines (those that close outside $R_{A}$ ); a magnetosphere results from the suppression of the mass loss at or near the magnetic equator. Any outflow will be governed by the magnetic field within the Alfvén radius so that the filling of a magnetosphere around the star is a necessary consequence of the combined effects of mass loss and trapping.

The circumstellar environment generated by a centrifugal wind looks like an isothermal atmosphere with a scale height given by the sound speed and the rotational frequency. This is because the only acceleration in the system varies as $r \Omega^{2}$ and the scale height is simply given by $a_{s}^{2} / g_{\text {eff }}$ (where $g_{\text {eff }}$ is the effective acceleration) so that $\rho(r)=\rho_{0} \exp \left(-\frac{r^{2} \Omega^{2}}{a_{0}^{2}}\right)$. Here $\rho_{0}$ is the density at the base of the dead zone. This is also important because it means that the trapped plasma will have very steep density gradients out to the edge of the magnetopause. If viewed in the absorption lines, this means that the rotationally generated line profiles will have "soft" terminal edges, not the steep saturation that comes from the turnover in the radiative acceleration-produced velocity of normal early type stellar winds. The structure of an oblique magnetosphere has been discussed by Sakurai (1986) but not including the effects of radiation pressure. However, in general, one would anticipate that the field beyond the Alfvén radius will form a current sheet that is dominated by a flopping mode in the rotational equator (Hill, Dessler, and Goertz 1983).

The stellar magnetosphere problem is quite different from that posed by pulsars or even planetary magnetospheres for several reasons. A pulsar is a neutron star with such a strong magnetic field and so high a rotational frequency that the Alfvén surface lies far outside of the light cylinder. Consequently, there is a unique outer boundary whose radius depends only on the rotation period of the star. The light cylinder is physically quite complicated because of inertial effects in the corotating plasma, but otherwise it is remarkably simple. Any flow out of the pulsar magnetosphere has to connect to infinity at $r=c \Omega$, but this is a unique radius. The potential drop between the rotational pole and equator is generated by the rotation and the field. Thus, the rate of filling of the magnetosphere is determined by the rotation. While this hasn't been completely solved, there is a basic understanding of how this process proceeds.

The planetary magnetosphere problem is also different from the stellar one because its boundary is set by pressure equilibrium with an external medium, the solar wind, and because it is filled from the outside (e.g. Moore and Waite 1988). There is much to learn from these structures that is relevant to the stellar environment, for instance for the AM Her stars, but many of the effects seen in planetary magnetospheres simply do not occur with stars. The Jovian magnetosphere, so far the best studied, displays much the same structure we can expect to find in a stellar oblique rotator. Outside of a few Jovian radii, the field shows a concentrated current sheet structured by a bending wave driven by the rotation of the oblique magnetic field. VLA imaging has even been able 
to show some of the details that we cannot resolve for the helium stars (see de Pater and Klein 1989).

What makes the upper main sequence stellar case so different is the interplay between the mass loss rate and the location of the Alfvén surface. Recall that the simplest estimate of the radius of the trapped plasma is when $v_{\text {rot }}=V_{A}$, that is when the corotation velocity is the same as the Alfvén speed. The physical problem is that $v_{A}$ depends on the local density at the Alfvén surface, which in turn depends on the mass loss rate. In other words, the more you load the magnetosphere with matter, the lower $v_{A}$ at the same field strength (the greater the effective inertia of the trapped plasma) and therefore the smaller the magnetosphere. The self-consistent structure of the stellar magnetosphere must be solved including the angular momentum of the star. Unlike the solar case, the radiative driving imposes a velocity gradient on the material very near the photosphere and, as Barker and Marlborough (1982) pointed out, this means that the radial velocity of the material isn't zero. In the Weber-Davis solution for an evaporative wind, this changes the inner boundary condition and consequently the angular momentum distribution through the entire circumstellar material.

There should be an obvious observational dependence of the magnetospheric properties on several photospheric parameters. In particular, in the hottest stars, where radiation pressure drives the mass loss, the presence or absence of a stellar wind should affect the magnetospheric plasma. In the cooler stars, the star's rotational velocity should determine the filling of the magnetosphere.

Finally, one of the most important effects of the magnetospheric plasma is to assist in spinning down the rotation rate of the star. Compared with the rate originally calculated by Deutsch, the confinement of matter out to $R_{A}$ is far more efficient at transferring angular momentum away from the star than Poynting flux. In effect, one is scaling the angular momentum loss to be:

$$
\frac{d J}{d t}=\dot{M} R_{A}^{2} \Omega
$$

so that the spindown time, $t_{s d}=J / \dot{J}$ is approximately given by:

$$
t_{s d}^{-1}=\frac{2}{3 K} \frac{\dot{M}}{M}\left(\frac{r_{A}}{r_{0}}\right)^{2} \approx \frac{\dot{M}^{2 / 3}}{M}\left(\frac{B_{0}}{\Omega}\right)^{2 / 3} .
$$

Here $K$ is the numerical factor for the central concentration needed for the moment of inertia of the star. Given the low mass loss rates expected for main sequence helium peculiar stars, however, this timescale is longer than the main sequence. However, a pre-main sequence magnetosphere can have a drastic effect on the rotation rate of the star if the lifetime of the high mass loss phase is long enough. This is expected in the lower mass Ap stars, but the rapid descent to the main sequence of the more massive helium weak and helium strong stars likely mitigates the action of this phase.

\section{WHAT ARE THE OBSERVABLES?}

Whether the star is rotating or not, the material streaming out of the magnetic poles will be able to escape the star more easily than that confined to the magnetic equator. Thus the flow will, at large distance, look jet-like. The 
field lines that close within the Alfvén point, however we estimate such a distance, will trap the outflow and permit the formation of a magnetosphere while those which are open at that distance will connect to infinity and permit free streaming. A critical magnetic latitude exists for the star, $\theta_{c}$, estimated by

$$
\theta_{c}=\sin ^{-1}\left(R_{A} / R_{0}\right)^{-1 / 2}
$$

for a dipole field which opens as the Alfvén point moves closer to the stellar surface. The magnetic field confines the escaping material in space and alters the amount of material at the terminal velocity. The trapped matter corotates (approximately) with the surface out to $R_{A}$, so one would expect a broad emission line with a width $\Omega R_{A}$ and an undisplaced narrow absorption component superimposed.

From the jet, seen against the photosphere, the profile should have a substantially reduced emission component (irrespective of the magnetospheric component) and a broad blueward extended absorption profile. In other words, the profile does not look anything like a normal $\mathrm{P}$ Cygni profile ( $c f$. Kunacz 1984). The trapped matter can have very large optical depth but may (depending on the magnetic field geometry) have a relatively low covering factor over the stellar surface. In general, we would expect static, very opaque material that has a scale height comparable with the stellar surface (for a dipole) and rotationally broadened out to the Alfvén radius. However, the material at this large distance is not very dense, and therefore the profile should have a shaded blue wing out to the terminal velocity.

When viewed edge-on, that is with the plasma intervening between the observer and the photosphere, a very strong resonance line should occur. When viewed orthogonally, that is face on, a weak emission line may appear. This will be combined with the jet absorption from the magnetic pole, as discussed by Shore and Brown (1990) (see also Brown et al. 1985). The UV thus provides the most obvious signature. Optical lines should generally show emission because of the reduced optical depth, and shell absorption should appear on the higher Balmer lines when the magnetosphere transits over the stellar disk. For systems that never display transits, only emission from the $\mathrm{H} \alpha$ and possibly $\mathrm{H} \beta$ lines should be observed.

In general, the Alfvén point will be at lower velocity than the escape speed. Consequently, a centrifugal wind will produce line profiles that are narrower than the polar jet, even taking the density distribution into account. Also, the line asymmetry depends on the obliquity and inclination of $\hat{\Omega}$ to the line of sight. The polar jets produce strong asymmetries, from both occultation and the effects of the phase function for scattering. The magnetosphere produces asymmetric lines also, but not as severe as the jet case.

\section{SUMMARY OF OBSERVATIONAL RESULTS: THE OPTICAL DATA}

Optical observations were the first indicators of the existence of magnetically controlled circumstellar plasma in the helium peculiar stars. Walborn showed that the three Orion association helium strong stars show time-variable $\mathrm{H} \alpha$ emission. The character of this emission is unusual. There are $V / R$ variations that are reminiscent of the Be stars, but the period of the variation is both short and remarkably stable (Pedersen 1979, Walborn 1983). The rotation periods 
of all of these stars are short as determined from both photometry and $\mathrm{He}$ I line variations, and the Balmer line emission varies on the same timescale. The emission line variations are repeatable over many thousands of rotations, indicating that the plasma clouds are quite well confined (however structured). There is also a curious relationship with the longitudinal magnetic field, at least for HD 37479 - the emission region seems to be maximized near the magnetic equator.

The same stars also display a dramatic change in the cores of the higher Balmer series and also in the last visible Balmer line, phenomena discovered by Groote and Hunger (1977). Narrow, shell-like cores develop on the higher series members essentially coincidentally with photometric minima for HD 37017 and HD 37479. Although the detailed behavior is not known for the other stars, it's likely that HD 64740 also shows this.

One of the most intriguing observations was by Kemp and Herman (1977), who reported the detection of linear polarization at $\mathrm{H} \alpha$ in $\sigma$ Ori E. The data had a large scatter, but the result was quite suggestive. This observation was not repeated, and this is a lacuna in our knowledge that must be filled. Polarization measurements of Balmer emission lines provide the only indication we can get of the geometry of the scattering region surrounding the star. Although the results were consistent with some sort of disk, or thin magnetosphere, the data were not of sufficient quality to warrant detailed models. Current technology could produce such necessary observations.

Bolton et al. (1987) have detailed the optical line variations in $\sigma$ Ori $\mathrm{E}$, arguing that the circumstellar plasma is distributed in several relatively narrow regions located some distance from the stellar surface. They base this on two properties of the $\mathrm{H} \alpha$ emission. The first is that it does not seem to extend to the rotational velocity of the $\operatorname{star}\left(v \sin i \approx 150 \mathrm{~km} \mathrm{~s}^{-1}\right.$ for this star). Then, the emission wing extends to about ten times the surface rotational velocity. The last is that the line profile variations are not continuous so on this basis the plasma is not uniformly distributed around the star. They also argue that the matter is located at the magnetic equator and that the highest density regions seem to be near the intersection points between the magnetic and rotational equator.

Landstreet and Borra (1978) and Hunger and Groote (1982) have interpreted this as the evidence for a magnetosphere in at least $\sigma$ Ori $\mathrm{E}$. Nakajima presented a phenomenological model for the $\mathrm{H} \alpha$ variations that is basically the same as that described by Groote and Hunger (1982). Hunger et al. (1990) have since argued that the geometry of this trapped plasmasphere is quite complex and very non-axisymmetric. While their geometry is more complicated, the basic picture described by Nakajima and Bolton et al. still holds - the circumstellar matter is confined by the magnetic field but may be structured by the rotation.

The helium weak stars do not show strong $\mathrm{H} \alpha$ variations ( $c f$. Takada-Hidai 1988). In a real sense, these stars, especially the $s n$ subgroup, look perfectly "normal" as far as the helium peculiar stars go. They show low amplitude spectrum variations of all of the usual species, like He I and Si II, and essentially no indication that anything is unusual about their circumstellar environments. There is a small group that seem to have the special property of displaying strong trapped circumstellar plasma. These stars all have been classified as sn stars based on their optical properties. Now this is very puzzling because the only 
feature that causes these stars to be morphologically distinguished is that they have C II $\lambda 4267 \AA$ lines that are too strong for their spectral types. However, they share one interesting property: their helium deficiencies are large enough to produce severe misclassification. They are called B8 III instead of B4 V, as their effective temperatures seem to indicate.

But the presence of magnetospheres isn't even a global property of the $s n$ helium weak stars! While only two others have been studied, there is no indication that all of the stars so labeled have strong circumstellar regions. If there is any mystery here it is what special property HD 5737,21699 , and 79158 possess that causes them to behave like the helium strong stars.

\section{SUMMARY OF OBSERVATIONAL RESULTS: THE ULTRAVIOLET DATA}

The helium weak stars don't generally show indications of magnetospheres. A survey by Brown et al. (1984), although more cursory than might now be possible, showed that only three of 14 helium weak and/or silicon stars show any evidence of C IV or Si IV stronger than would be expected for the stellar temperature. The three stars that show variable resonance lines, HD 5737, 21699 , and 79158 , are each quite individual (Shore et al. 1990). The variations of HD 21699 have been discussed by Brown et al. (1985) and Shore et al. (1987). The predominant C IV profile variation seems to be due to polar outflow. The line profile becomes quite asymmetric, with an extended blue wing, but it never displays a P Cyg profile. There is never a phase when the strong C IV is completely absent, and the strongest absorption coincides with the magnetic pole. This is important because only one pole, the positive one, coincides with the strong line phase. HD 5737 and HD 79158 show almost identical variations. The strongest absorption lines correspond to magnetic equatorial transits.

The helium strong star HD 37479 has been singled out in several discussions, especially by Hunger (this conference). He argues that the variations in HD 37479 are a NLTE effect and that the C IV profile variations are connected with atmospheric structure. In one sense this is certainly true - the low density and relatively high temperature of the circumstellar gas are ideal for all kinds of departures from LTE to occur. This is actually an excellent way to understand what is happening. The variations of the circumstellar lines must also include, for the helium strong stars, a proper accounting for that component that may be due to photospheric variations of the resonance lines. However, in another sense this separate place accorded $\sigma$ Ori $\mathrm{E}$ is wrong. The phenomena are completely general among the helium strong upper main sequence stars, regardless of temperature, and they extend almost identically into the helium weak domain with the $s n$ stars. The problem is, in a sense, that concentrating on the one star that is best known leads to the incorrect perception that HD 37479 is unique. It isn't.

The helium strong stars, because of their relatively larger number, provide the key to the interpretation of the UV resonance line variations (Shore and Brown 1990). There is reason to believe that the bulk of the sample of helium strong stars are rapid rotators. Therefore, the slow rotators are probably simply seen nearly pole-on. Thus the aligned systems, where the magnetic field, $\hat{\mathbf{B}}$ is parallel to the rotation axis, $\hat{\Omega}$, should be seen constantly in emission due to scattering. Those stars that have $\hat{\mathbf{B}}$ perpendicular to $\hat{\Omega}$ should show only strong, constant, 
absorption lines. In both cases, the line formation is assumed to be mainly due to scattering. The asymmetry that is seen in several emission stars, notably HD 96446 and HD 58260 may be due to the combined effects of absorption by the jet and emission by the magnetosphere (Shore and Brown (1990) show a simple decomposition of such a profile). The other systems, with intermediate inclinations, will show variable profiles that should range from strong absorption to weak emission, depending on aspect angle (for examples see Shore and Brown 1990).

One system shows large scatter and perhaps even intrinsic variability - HD 37776 , the only dominantly quadrupolar system among the helium strong stars. Otherwise the profiles look much the same as for the other stars. While it is clear that virtually all of the helium stars have some higher order moments in their fields, this star is the most extreme.

\section{SOME UNSOLVED PROBLEMS}

The key problem still facing our understanding of the circumstellar environment is why only some of the helium weak stars show trapped plasma. There doesn't seem to be any obvious answer to this, even after several large-scale surveys and considerable work in both the optical and other wavelength regions. First, the existence of a magnetosphere does not obviously depend on rotation frequency. $\alpha \mathrm{Scl}$ has almost an order of magnitude lower $\Omega$ than HD 21699 or 36 Lyn, yet it has a fully developed stable magnetosphere. There is no substantive difference between the plasma confinement characteristics around the helium rich and helium weak stars. This in spite of their very different temperatures and rotation frequencies, not to say radii and magnetic field strengths.

The magnetospheric structure doesn't even seem to depend strongly on the surface magnetic field strength. However, it is entirely possible that the surface field configuration, that is the multipolarity, may come into play. It is possible that the presence of a large scale quadrupole component, so evident in HD 37776 , leads to a less stable magnetospheric region.

One helium strong star is a real puzzle - HD 184927. Here all of the phenomenology that is seen in the intermediate obliquity helium strong stars is observed. However, the rotation period of this star seems to be long, about 9.5 days (Bond 1978). Again like the $s n$ stars, this may mean that there isn't really a strong dependence of the magnetospheric properties on the rotation frequency. HD 184927 may indeed stand to the helium strong stars as $\alpha$ Scl does to the helium weak stars. But why?

The radio data, discussed at this conference by Linsky, show that none of the helium weak magnetospheric stars has detectable radio emission while most of the helium strong stars do. The limits, however, are not seriously constraining and they may yet prove to be radio emitters. There are several ways of generating the energetic particles required for this emission, which appears to be nonthermal (Leone 1992, preprint; Linsky et al. 1992, see Linsky's talk at this meeting). One is to accelerate particles in the interface between open and closed field lines via a Kelvin-Helmholtz instability. This will be excited when the flow speed exceeds the Alfvén speed, something that requires the active driving of a strong stellar wind near terminal speed. The helium strong stars certainly are capable of supporting such a wind, and there are indications from the UV line 
profiles that several of them show it. But for the helium weak stars this isn't possible since they aren't expected to show strong outflows or large velocities. An alternative mechanism is to imagine that the magnetosphere forms an oscillating current sheet at large distance (see Dessler 1983). Beyond the Alfvén radius, the magnetic field of an oblique rotator is a free oscillator that, because it is loaded with mass, forms an unstable current sheet. This sheet may be subject to a tearing mode instability that could be the source of energetic particles. This is the sort of picture that Linsky et al. (1992) have in mind. Reconnection mechanisms have been discussed by Havnes and Goertz (1984), for instance, that may also remove considerable mass from the magnetosphere (see also Linker et al. 1992).

Given the current (high) upper limits for any of the non-detections, the radio emission is probably of secondary importance in diagnosing what is happening in these stars. It is vital for showing that the environments around the helium strong stars are populated by thermalized and superthermal particles. This will certainly have an effect on the ionization balance. On the other hand, the thermal plasma, while hot, is still the predominant component.

$\underline{\text { CODA }}$

It would be wonderful if some of our colleagues in other fields, like space physics and pulsars, could be induced to join in the effort of understanding these stars. But clearly, for quite some time to come, there's more than enough work for everyone.

\section{ACKNOWLEDGMENTS}

I thank Helmut Abt, Dave Bohlender, Tom Bolton, Doug Brown, Steve Drake, Alex Fullerton, Tim Hankins, Ivan Hubeny, Kurt Hunger, John Landstreet, Myron Smith, and George Sonneborn for stimulating discussions. This work was supported in part by NASA. I especially thank Mike Dworetsky for his patience during the preparation of this manuscript.

\section{REFERENCES}

Barker, P. K. and Marlborough, J. M. 1982, ApJ, 254, 297.

Belcher, J. and MacGregor, K. 1976, ApJ, 210, 498.

Bolton, C. T., Fullerton, A. W., Bohlender, D., Landstreet, J. D., and Gies, D. R. 1987, Physics of Be Stars (eds. A. Slettebak and T. P. Snow) (Cambridge: Cambridge Univ. Press) p. 82.

Bond, H. E. 1978, PASP, 88, 905.

Brown, D. N., Shore, S. N., Barker, P. K. and Sonneborn, G. 1984, in The Future of Ultraviolet Astronomy Based on Six Years of IUE Research (eds. Mead, J., Chapman, R. D. and Kondo, Y.) (NASA CP-2349), p. 487.

Brown, D. N., Shore, S. N. and Sonneborn, G. 1985, $A J$, 80, 1354.

de Pater, I. and Klein, M. J. 1989, in Time-Variable Phenomena in the Jovian System (NASA SP-494), p. 139.

Dessler, A. J. (ed.) 1983, Physics of the Jovian Magnetosphere (Cambridge: Cambridge Univ. Press). 
Drake, S. A., Abbott, D. A., Bastien, T. S., Bieging, J. H., Churchwell, E., Dulk, G., and Linsky, J. L. 1987, ApJ, 322, 902.

Goldreich, P. and Julian, W. 1969, $A p J, 157,869$.

Groote, D. and Hunger, K. 1976, $A \& A, 52,303$.

Groote, D. and Hunger, K. 1977, $A \mathscr{E A}$, 56, 129.

Groote, D. and Hunger, K. 1982, $A \mathcal{G} A, 116,64$.

Havnes, O. and Goertz, C. K. 1984, $A \mathscr{E A}, 138,421$.

Hesser, J. E., Walborn, N. R., and Ugarte, P. 1976, Nature, 262, 116.

Hill, T. W., Dessler, A. J., and Goertz, C. K. 1983, in Physics of the Jovian Magnetosphere (ed. A. J. Dessler) (Cambridge: Cambridge Univ. Press) p. 353.

Hunger, K., Heber, U., and Groote, D. 1990, in Properties of Hot Luminous Stars (ed. C. Garmany) (San Francisco: ASP Press), p. 307.

Hunger, K. 1992, these proceedings.

Kemp, J. C. and Herman, L. C. 1977, ApJ, 218, 770.

Kunacz, P. 1984, ApJ, 276, 677.

Landstreet, J. D. and Borra, E. F. 1978, ApJL, 224, L5.

Linker, J. A., van Hoven, G., and McComas, D. J. 1992, JGR, 97(A9), 13733.

Mestel, L. 1968,MNRAS, 138, 359.

Michel, F. C. 1991, Theory of Pulsar Magnetospheres (Chicago: Univ. of Chicago Press).

Moore, T. E. and Waite, J. H. Jr. (eds.) 1988, Modeling Magnetospheric Plasma (Washington: AGU Geophysical Monograph 44).

Nakajima, R. 1985, Ap. Space Sci., 116, 285.

Nerney, S. and Suess, S. 1987, ApJ, 321, 355.

Pacini, F. 1967, Nature, 216, 567.

Phillips, R. B. and Lestrade, J.-F. 1988, Nature, 334, 329.

Pedersen, H. 1979, $A \& A S, 35313$.

Sakurai, T. 1986, $A \mathscr{E} A, \mathbf{1 5 2}, 121$.

Shore, S. N. 1987, $A J, 94,731$.

Shore, S. N. 1990, in Evolution in Astrophysics: IUE in the Era of New Space Missions (ed. E. Rolfs) (ESA SP-316).

Shore, S. N., and Brown, D. N. 1990, ApJ, 365, 665.

Shore, S. N., Brown, D. N. and Sonneborn, G. 1987, AJ, 94737.

Shore, S. N., Brown, D. N., Sonneborn, G., Landstreet, J. D., and Bohlender, D. A. 1990, ApJ, 348, 242.

Takada-Hidai, M. 1988, PASP, 88, 146.

Walborn, N. R. 1974, ApJL, 191, L95.

Walborn, N. R. 1983, ApJ, 268, 195.

Walborn, N. R. and Hesser, J. E. 1976, $A p J L, 205,87$.

Weber, E. J. and Davis, L. Jr. 1967, ApJ, 148, 271. 